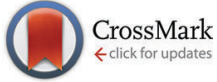

Cite this: J. Mater. Chem. C, 2017, 5, 2591

\section{Ultra-broadband optical amplification at telecommunication wavelengths achieved by bismuth-activated lead iodide perovskites $\dagger$}

\author{
Yang Zhou, ${ }^{a}$ Zi-Jun Yong, ${ }^{a}$ Wei Zhang, ${ }^{\mathrm{b}} \mathrm{Ju}$-Ping Ma, ${ }^{a}$ Aditya Sadhanala, ${ }^{\mathrm{c}}$ \\ Ya-Meng Chen, ${ }^{\mathrm{a}}$ Bo-Mei Liu, ${ }^{\mathrm{a}}$ Yi Zhou, ${ }^{\mathrm{a}}$ Bo Song ${ }^{\mathrm{a}}$ and Hong-Tao Sun*a
}

It is extremely difficult to achieve hybrid halide perovskite semiconductors with luminescence at wavelengths longer than $1.0 \mu \mathrm{m}$, because of the inherent limitation of their band gaps. We show herein that solution-processable, Bi-activated, high-quality $\mathrm{MAPbl}_{3}$ films can be adopted as a new gain medium operating in the whole telecommunication window of 1260-1625 nm. Additionally, the structural and optical properties of $\mathrm{Bi}$ doped $\mathrm{MAPbl}_{3}$ have been investigated. The results indicate that the NIR PL originates from the structural defects induced by Bi. Finally, we accomplished optical amplification in the whole telecommunication window by using $\mathrm{Bi}$-doped $\mathrm{MAPb}_{3}$ films, which represents the first work where such a performance is attained among lead halide perovskites and Bi-doped photonic films. This work opens up exciting possibilities of using perovskite semiconducting materials as gain media for optical amplifiers and lasers operating in the telecommunication window.
Received 22nd December 2016, Accepted 14th February 2017

DOI: $10.1039 / \mathrm{c} 6 \mathrm{tc05539g}$

rsc.li/materials-c as a universal requirement for the successful use of the whole optical communication window available in silica fibres. ${ }^{4}$

During recent years, solution-processable metal halide perovskite semiconductors, in particular hybrid organic-inorganic lead halide perovskites, have shown huge potential in the field of optoelectronic devices including solar cells, ${ }^{5-8}$ light-emitting diodes, ${ }^{9-14}$ lasers, ${ }^{15-19}$ photodetectors, ${ }^{20,21}$ and single photon sources. $^{22}$ This has mainly been driven by their low-cost solution processability and outstanding intrinsic optoelectronic properties (e.g., large absorption coefficients, long carrier lifetimes, and tunable band gaps). ${ }^{23-28}$ So far, $\mathrm{MAPbX}_{3}$ (where $\mathrm{MA}=$ methylammonium and $\mathrm{X}=\mathrm{Cl}$, Br or I, or mixtures thereof) semiconductors have enabled inexpensive solar cells with certified power conversion efficiencies exceeding $22 \%,{ }^{28}$ and efficient lasing and amplified spontaneous emission, operating in the spectral range of 400-800 nm, from thin films or nanostructures have been demonstrated. Although pristine metal halide perovskite solid solutions offer considerable tunability of the optical band gap by integrating different halides or cations into crystal structures, it is extremely difficult to endow them with luminesce at wavelengths longer than $1.0 \mu \mathrm{m}$, because of the inherent limitation of their band gaps, thus greatly limiting their applications in telecommunications. At present, strategies available for engineering halide perovskites luminescing at longer wavelengths are rather limited. Ning et al. reported an approach for in situ epitaxial growth of perovskites on quantum-dot surfaces, and the resulting quantum-dot-inperovskite nanocomposite displays photoluminescence (PL) and electroluminescence (EL) in the range longer than $1.0 \mu \mathrm{m}$ owing 
to the band-edge emission of PbS quantum dots. ${ }^{14}$ Doping of semiconductors with foreign ions is a powerful route to tune their photophysical and electrical properties. ${ }^{29,30}$ Very recently, we demonstrated that ultrabroad near-infrared (NIR) EL covering the whole telecommunication windows is achievable using bismuthdoped $\mathrm{MAPbX}_{3}$, as a result of the formation of new luminescent defects. ${ }^{30}$ However, the incomplete film coverage, coupled with the not fully understood luminescence mechanism, to some extent, hampers its prospects for practical applications. Designing NIR-luminescent, high-quality, Bi-doped perovskites is therefore of considerable interest, since it would provide opportunities for constructing high-performance optoelectronic devices.

Here, we demonstrate that solution-processable, Bi-activated, high-quality $\mathrm{MAPbI}_{3}$ films can be used as a new gain medium operating in the whole telecommunication window. The fabrication of pinhole-free, Bi-activated, high-quality $\mathrm{MAPbI}_{3}$ films can be achieved by an alternative, optimized solution-processable strategy. The as-prepared film features ultrabroad NIR PL covering the whole telecommunication window with significantly improved film quality in comparison to that fabricated via a method reported previously. The result gleaned from photothermal deflection spectroscopy (PDS) measurement provides a direct indication that the NIR PL originates from the structural defects induced by Bi doping. Additionally, we investigate the properties by steady-state and time-resolved PL spectroscopy at room and cryogenic temperatures, which further deepens our understanding of the photophysical characteristics for such a doped perovskite system. Finally, we show that this emerging system can be employed to achieve optical amplication in the whole telecommnication window, pumped at very low power densities. This is, as far as we know, the first work on the attainment of such a performance among lead-halide perovskite semiconductors and Bi-activated NIR-luminescent films. Our findings suggest that Bi-activated perovskite systems are promising gain media for NIR photonics.

\section{Experimental}

\section{Fabrication of Bi-doped $\mathrm{MAPbI}_{3}$ films}

Lead acetate (99.999\%, Acros), $\mathrm{PbI}_{2}$ (99.9985\%, Alfa), $\mathrm{BiI}_{3}$ (99.99\%, Aladdin) and DMF (99.8\%, Acros) were used without further purification. $\mathrm{CH}_{3} \mathrm{NH}_{3} \mathrm{I}$ was purchased from Xi'an Polymer Light Technology Corp. The perovskite precursor solution was prepared by mixing MAI and $\mathrm{Pb}\left(\mathrm{CH}_{3} \mathrm{CO}_{2}\right)_{2}$ in a 3:1 molar ratio in DMF, which was stirred for $5 \mathrm{~h}$ at room temperature in a nitrogen-filled glovebox ( 0.1 ppm $\mathrm{H}_{2} \mathrm{O} ; 0.1 \mathrm{ppm}_{2}$ ) to obtain a $0.8 \mathrm{M}$ solution. Additional $\mathrm{BiI}_{3}$ in $\mathrm{DMF}$ was added to the mixture to achieve $0.01 \%$ or $0.05 \%$ doping, finally obtaining a solution that was then filtered and spin-coated at $3000 \mathrm{rpm}$ for $45 \mathrm{~s}$ to give a film. The film was thermally treated at $100{ }^{\circ} \mathrm{C}$ for $5 \mathrm{~min}$. As a comparison, we also fabricated a $\mathrm{PbI}_{2}$-derived film (MAI and $\mathrm{PbI}_{2}$ in a $1: 1$ molar ratio) by using a previously reported method. ${ }^{30}$

\section{Characterization}

XRD patterns were taken at room temperature using a Bruker D8 ADVANCE diffractometer with $\mathrm{Cu} \mathrm{K} \alpha$ radiation $(\lambda=1.54056 \AA)$.
Scanning electron microscopy (SEM) images were acquired on an S-4700 microscope (Hitachi, Japan) operating at $15 \mathrm{kV}$. The chemical composition of the $\mathrm{PbAc}_{2}$-derived film was investigated using a scanning electron microscope (SEM; ZEISS EVO-18) equipped with an energy-dispersive X-ray analyzer. AFM images were obtained using a Multimode 8 microscope (Bruker, Santa Barbara, CA) with a peak force quantitative nanomechanical mode in air and scanning over a range of $15 \mu \mathrm{m}$ by $15 \mu \mathrm{m}$ at a resolution of $256 \times 256$ data points. The surface roughness was measured as the root mean-squared roughness over the scanning area. PDS was performed using a similar method to that previously reported by Zhang et al. ${ }^{8}$ The PL spectra shown in Fig. 1b was analyzed with an FLS 980 spectrofluorometer (Edinburgh Instruments Ltd) equipped with a liquid $\mathrm{N}_{2}$ cooled PMT (Hamamatsu, R5509-73). All other PL spectra were taken by using a monochromator (Horiba, iHR550) equipped with a photomultiplier (PMT) (Hamamatsu, R928) and an electricallycooled PMT (Hamamatsu, H10330-75). The spectral response of the detection system was corrected using a standard sample. Note that owing to the difference in spectral response for different PMTs, the spectral range of PL taken is different in some cases. The internal PL QEs at room temperature were measured by a previously reported method, ${ }^{30}$ using a $730 \mathrm{~nm}$ semiconductor laser as the excitation source. The integrated PL intensity ratio of $I_{\text {cryo }}$ to $I_{296 \mathrm{~K}}$ was used to roughly estimate the internal QE at cryogenic temperatures based on the following equation, $\mathrm{QE}_{\text {cryo }}=\mathrm{QE}_{296 \mathrm{~K}} \times I_{\text {cryo }} / I_{296 \mathrm{~K}}$, where $I_{\text {cryo }}$ and $I_{296}$ are PL intensities taken at low temperatures and $296 \mathrm{~K}$, respectively. The temperature-dependent PL spectra were taken by using a liquid helium cooled cryostat. The time-resolved PL measurements at different temperatures, under the excitation of nanosecond-pulsed light from the second harmonic $(532 \mathrm{~nm}, 10 \mathrm{~Hz})$ of a Nd:YAG laser, were performed by detecting the modulated luminescence signal with a PMT (Hamamatsu, H10330-75), and then analyzing the signal with a photon-counting multichannel scaler. A pump and probe transmission technique was used for the net gain measurements. ${ }^{31}$ The pump and probe beams were provided by a $450 \mathrm{~nm}$ laser diode and a lamp (Ocean Optics DH-3-BALCALEXT), respectively, which were imaged to a spot with a $2 \mathrm{~mm}$ diameter on the sample surface. During the measurement, the film was kept in a closed-cycle He cryostat. The formula, $I_{\text {trans }}=I_{\text {inci }} \times$ $\mathrm{e}^{[(g-\alpha) d]}$, was used to deduce the material gain, where $I_{\text {trans }}, I_{\text {inci }}$,
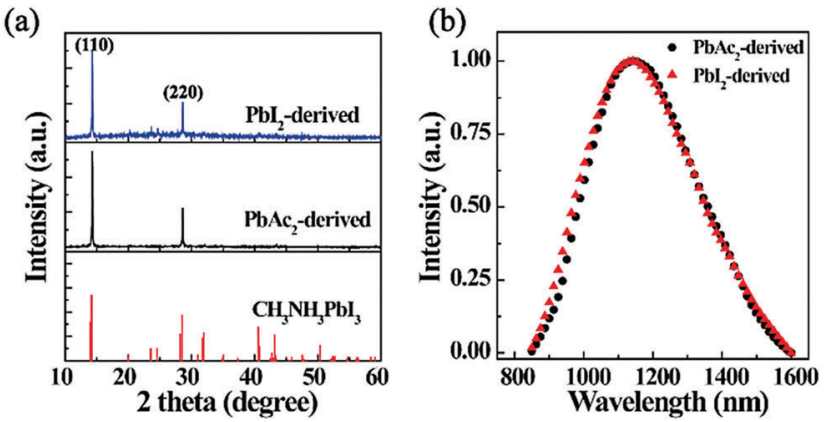

Fig. 1 (a) XRD patterns and (b) PL spectra of the $\mathrm{PbAc}_{2}$-derived and $\mathrm{Pbl}_{2}$ derived films under the excitation of $407 \mathrm{~nm}$. 
$(g-\alpha)$, and $d$ are the transmitted probe beam with pumping, the original incident probe beam without sample, net material gain, and film thickness, respectively. ${ }^{31}$

\section{Results and discussion}

The perovskite film was fabricated by spin-coating the precursor solution with a desired mole ratio by using $\mathrm{Pb}\left(\mathrm{CH}_{3} \mathrm{CO}_{2}\right)_{2}$ as the lead source under a $\mathrm{N}_{2}$ atmosphere in a glovebox (referred to as " $\mathrm{PbAc}_{2}$-derived film" hereafter), followed by thermal treatment at $100{ }^{\circ} \mathrm{C}$ for $5 \mathrm{~min}$ (see details in the Experimental section). The measured composition of the resultant film was found to have a molar ratio of $c a$. 1:3 for $\mathrm{Pb}: \mathrm{I}$ by energy dispersive X-ray spectroscopy (Fig. S1, ESI $\dagger$ ). The absence of detectable acetate in the final product indicates that the acetate is almost evaporated during the thermal treatment, which is consistent with the pristine film prepared by this strategy. ${ }^{8}$ For comparison, we also fabricated a film by using a previously reported method (referred to as the " $\mathrm{PbI}_{2}$-derived film" hereafter). ${ }^{30}$ The main difference between these two methods is the changes of lead source and stoichiometry of the precursors (see details in the Experimental section). In Fig. 1a, we compared the X-ray diffraction (XRD) patterns of films prepared by these two methods. It is noted that both techniques yield the same dominant diffraction peaks assigned to the crystallographic plane (110), indicating their identical orientation. It is noted that no fingerprints ascribed to Bi-related phases are detected, suggesting that $\mathrm{Bi}$ ions are incorporated into the perovskite films. Both films show ultrabroad NIR PL with nearly identical emission lineshapes under the excitation of $407 \mathrm{~nm}$, evidencing that the same class of active center contributes to such emissions (Fig. 1b). The top-view scanning electron microscope (SEM) images highlight the considerable differences between the film morphologies produced by the two methods (Fig. 2a and b). The $\mathrm{PbAc}_{2}$-derived film is extremely uniform and pinhole-free, appearing to have crystalline features on the length scale of hundreds of nanometres. In marked contrast, the $\mathrm{PbI}_{2}$ derived film appears to partially coat the substrate; obvious voids appear to extend directly to the substrate. We further evaluated the surface quality of the $\mathrm{PbAc}_{2}$-derived and $\mathrm{PbI}_{2}$-derived films using atomic force microscopy (AFM) (Fig. 2c and d). We calculated the root mean-squared roughness of the $\mathrm{PbAc}_{2}$-derived and $\mathrm{PbI}_{2}$-derived perovskite films to be 19.3 and $98.5 \mathrm{~nm}$, respectively, for areas of $15 \mu \mathrm{m} \times 15 \mu \mathrm{m}$. The roughness of the $\mathrm{PbAc}_{2}$-derived film is greatly reduced compared with that of the $\mathrm{PbI}_{2}$-derived film, as evidenced by both AFM line segments (Fig. 2e) and height distribution analysis (Fig. 2f). All these results evidence that using $\mathrm{Pb}\left(\mathrm{CH}_{3} \mathrm{CO}_{2}\right)_{2}$ as the lead source yields films with high quality in terms of film coverage and uniformity. This is because the byproduct $\left(\mathrm{CH}_{3} \mathrm{NH}_{3} \mathrm{Ac}\right)$ is thermally unstable and much easier to be removed than $\mathrm{CH}_{3} \mathrm{NH}_{3} \mathrm{I}$ at the same annealing temperature. This leads to an increased nucleation density, resulting in the formation of a uniform film with a large amount of relatively small crystals in a short time. ${ }^{8}$ We then examined the photophysical properties of the $\mathrm{PbAc}_{2}$-derived film by using a series of spectroscopic techniques. (a)

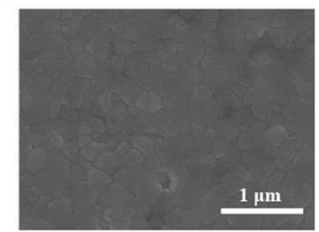

(c)

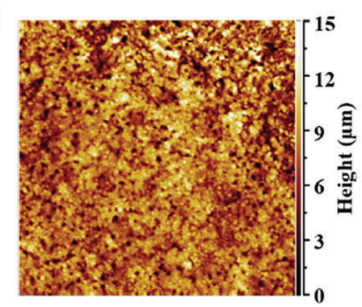

(e)

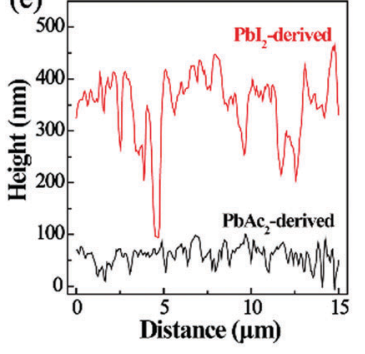

(b)

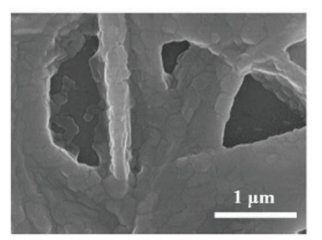

(d)

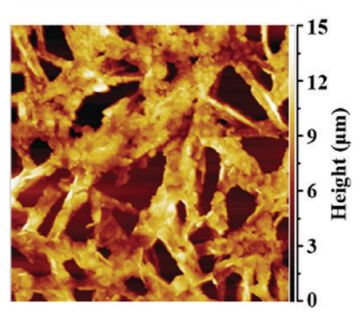

(f)

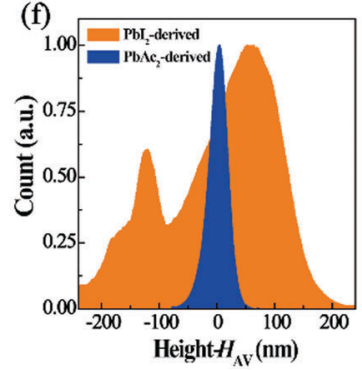

Fig. 2 Top-view SEM images of (a) $\mathrm{PbAc}_{2}$-derived and (b) $\mathrm{Pbl}_{2}$-derived films. AFM images of (c) $\mathrm{PbAc}_{2}$-derived and (d) $\mathrm{Pbl}_{2}$-derived films. AFM line segments (e) and AFM height distribution analysis ( $f$ ) of the $\mathrm{PbAc}_{2}$ and $\mathrm{Pbl}_{2}-$ derived films.

As is well known, the steepness of the optical absorption edge gives an indication of the quality of the material. The extent of the absorption tail below the band gap is intimately associated with the degree of energetic disorder within the material, which stems from thermal fluctuation of the ions comprising the material or from defects in the structure. PDS is an ultrasensitive absorption measurement technique that detects heating of the sample due to the non-radiative relaxation of absorbed light and is insensitive to reflection and scattering losses. This enables PDS to measure absorption with high sensitivity and a dynamic range of 4-5 orders of magnitude, which thus can be used to estimate an empirical energetic disorder parameter for the given semiconductor, known as Urbach energy, $E_{\mathrm{u}}{ }^{8,9,17} E_{\mathrm{u}}$ is given by $A(E) \propto \mathrm{e}^{E /\left(E_{\mathrm{u}}\right)}$, where $A$ is the absorbance and $E$ is the excitation energy in electronvolts. We took the PDS spectra of $\mathrm{PbAc}_{2}$-derived pristine and doped films to evaluate the influence of $\mathrm{Bi}$ incorporation on the electronic disorder of the systems. Interestingly, we find that $\mathrm{Bi}$ incorporation influences the sharpness of the absorption edge with respect to that of the pristine one. The linear fit to the Urbach tails in the absorption spectra for these systems is shown in Fig. 3. The fitted values are shown in Fig. 3, along with the respective fitting error. Within the measurement error, the Urbach energy is increased from 14.5 to $17 \mathrm{meV}$ as the $\mathrm{Bi} / \mathrm{Pb}$ ratio increases from $0.01 \%$ to $0.05 \%$, which are larger than $14.0 \mathrm{meV}$ for the undoped one. This strongly implies that $\mathrm{Bi}$ incorporation into the perovskite matrices leads to the creation of more defects (i.e., energetic disorder), even when the doping level is extremely low. Assuming the same level of thermal disorder in these films, we could ascribe 


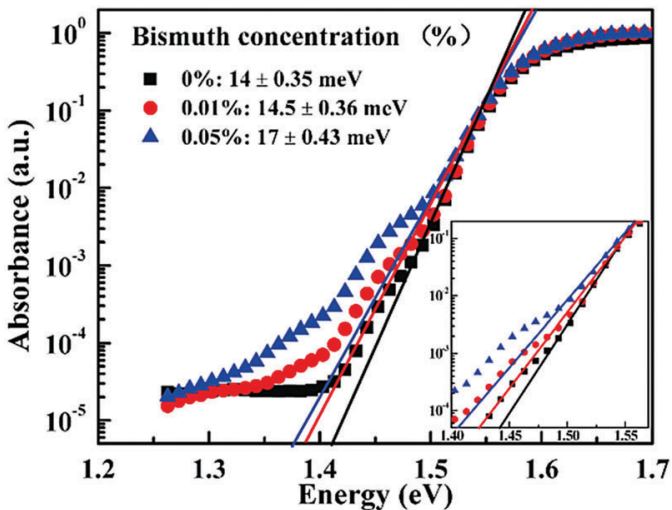

Fig. 3 PDS spectra of pristine, $0.01 \%$ and $0.05 \% \mathrm{Bi}$-doped $\mathrm{PbAc}_{2}$-derived films. Inset shows the enlarged PDS spectra from 1.4 to $1.57 \mathrm{eV}$. The lines in each plot are the linear fits to the Urbach tail used to calculate the Urbach energy. The fitted Urbach energy and fitting error are indicated for each sample. The fitting was not done below $1.5 \mathrm{eV}$ because those states despite being present below the bandgap have a density that is $3-4$ orders of magnitude lower than that at the band-edge and the Urbach energy is mainly influenced by the states closer to the band-edge that have a high density of states.

the increased Urbach energy to the increased concentration of structural defects induced by Bi doping. Note that the increased concentration of structural defects is accompanied by the appearance of NIR PL. This further verifies that the NIR PL is potentially connected with structural defects in Bi-doped films. The states below $1.5 \mathrm{eV}$ in the PDS spectra are directly correlated with the Bi content in the thin films and can be viewed as creation of new sub-bandgap states below the bandgap similar to the optically emissive states that are created in the IR region. Additionally, it is noteworthy that no obvious absorption band can be detected in the wavelengths longer than $1.0 \mu \mathrm{m}$ for Bi-doped systems in comparison to the pristine one (Fig. S2, ESI $\dagger$ ), meaning that the absorption coefficient of such NIR-luminescent defects is rather small.

To acquire more details of the photophysical properties of Bi-doped $\mathrm{PbAc}_{2}$-derived films, we took temperature-dependent steady-state PL measurements. In Fig. 4a, we show a contour map of the normalized temperature-dependent PL spectra of the Bi-doped $\mathrm{PbAc}_{2}$-derived film in the spectral range of $720-850 \mathrm{~nm}$. Fig. $4 \mathrm{~b}$ shows typical snapshots from this plot taken at different temperatures. The PL is centered at $772 \mathrm{~nm}$ at room temperature (denoted as "red PL"), and redshifts continuously until $140 \mathrm{~K}$, accompanied by a reduction of full width at half maximum (FWHM) (Fig. S3, ESI $\dagger$ ). Such a redshift is in good agreement with the temperature-dependent shift of the absorption edge of the $\mathrm{MAPbI}_{3}$ film, suggesting that the red PL is from a band-edge transition. ${ }^{27}$ Further decrease of temperature from 140 to $80 \mathrm{~K}$ results in a blueshift of PL. Furthermore, at $80 \mathrm{~K}$ a new shoulder appears at $c a .750 \mathrm{~nm}$, and becomes more evident as the temperature decreases to $10 \mathrm{~K}$, which can be ascribed to the emission from the free excitonic level. ${ }^{27}$ In Fig. 4c, we show a contour map of the normalized temperature-dependent NIR PL spectra of the Bi-doped $\mathrm{PbAc}_{2}$-derived film obtained under the excitation of $407 \mathrm{~nm}$ light with a power of $8 \mathrm{~mW}$. The room-temperature PL spectrum is centered at $1145 \mathrm{~nm}$, and no significant change in
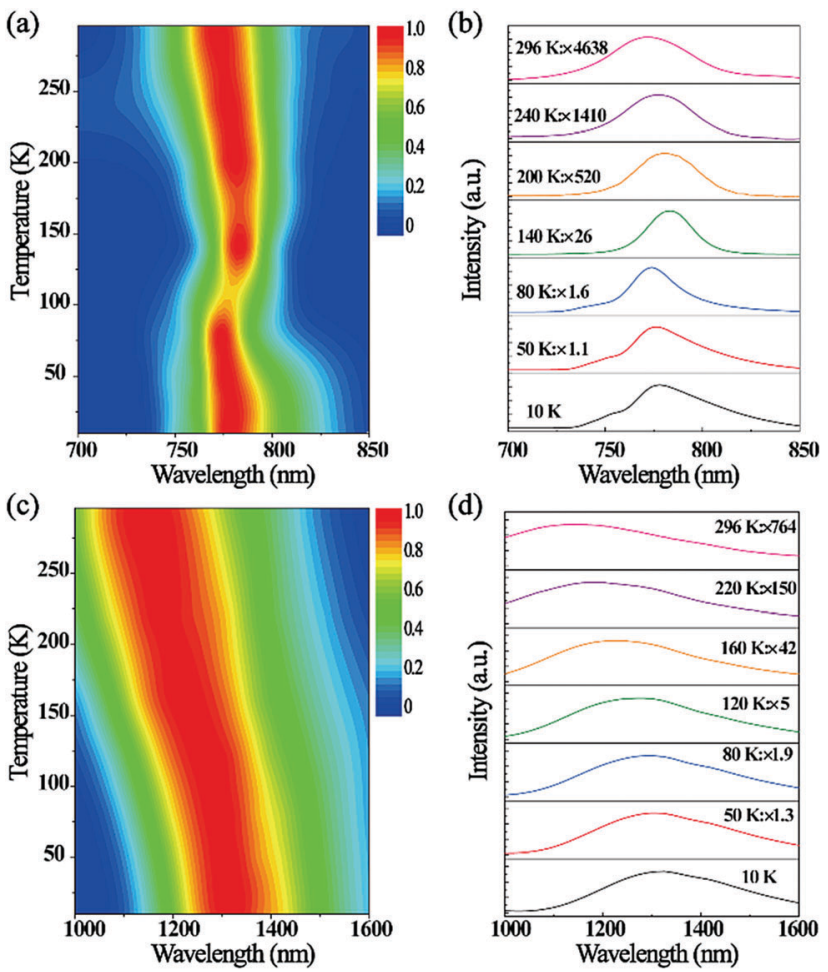

Fig. 4 Temperature dependent PL for the 0.01\% Bi-doped $\mathrm{PbAc}_{2}$-derived film. (a and c) Contour plot of PL spectra of the $\mathrm{PbAc}_{2}$-derived film at different temperatures under the excitation of $407 \mathrm{~nm}$ light. (b and d) Normalized $\mathrm{PL}$ spectra at different temperatures.

the lineshape is observed when the temperature is over $200 \mathrm{~K}$; further decreasing the temperature results in a redshift and a reduction of the FWHM of the emission (Fig. S4, ESI $\dagger$ ), as a consequence of weakened vibronic coupling between the active center and lattices. Additionally, the PL intensity monotonously increases with a decrease in temperature (Fig. 4d); the PL intensity at $10 \mathrm{~K}$ is over 700 times stronger than that at $296 \mathrm{~K}$, whereas it is around 5 times of that at $120 \mathrm{~K}$, strongly suggesting that a relatively low temperature (e.g., $120 \mathrm{~K}$ or liquid nitrogen temperature) is enough to suppress most of non-radiative pathways. This is of vital importance for engineering this system for practical applications, given the fact that such a low temperature can be readily achieved by using low-cost refrigeration units. Time-resolved PL dynamics measurements at different temperatures using nanosecond-pulsed photoexcitation clearly show that the decay becomes slower at low temperatures (Fig. S5, ESI $\dagger$ ), with an average lifetime of $c a .76 \mathrm{~ns}$ at $10 \mathrm{~K}$ (Table S1, ESI + ). Based on the results described above, we can conclude that Bi-doped $\mathrm{PbAc}_{2}$-derived films possess higher capability to convert the absorbed photons to NIR emissions at low temperatures.

In light of the tantalizing PL property of this system, we next employed it as a gain medium for light amplification in the NIR region. The most direct experimental evidence of light amplification from our system was provided by pump and probe transmission measurements. The experimental setup is shown in Fig. 5 a. A laser beam (pump) at $450 \mathrm{~nm}$ from a laser diode excites the sample in order to reach the population inversion required 
(a)

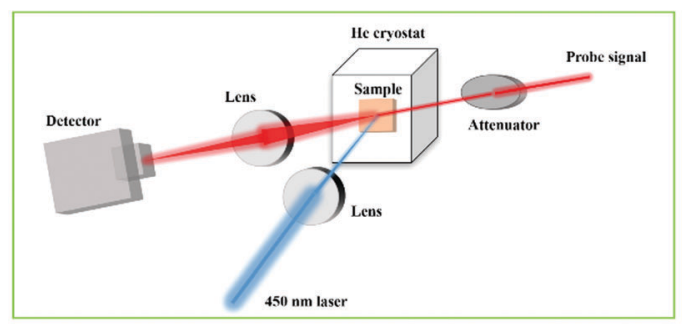

(b)

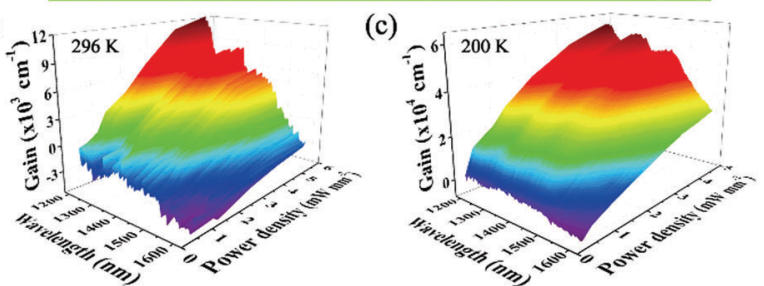

(d)

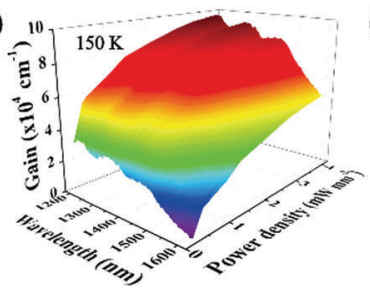

(e)

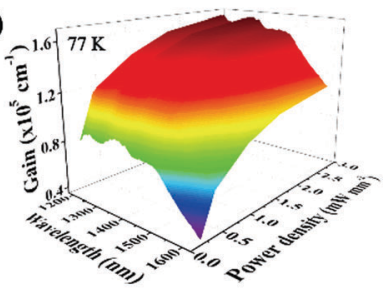

Fig. 5 (a) Schematic diagram of the experimental setup for the light amplification experiment. Gain measurements of the $0.01 \% \mathrm{Bi}$-doped $\mathrm{PbAc}_{2}$-derived film at different temperatures, (b) $296 \mathrm{~K}$, (c) $200 \mathrm{~K}$, (d) $150 \mathrm{~K}$ and (e) $77 \mathrm{~K}$.

for amplification, while a weak probe signal passes through the active layer with a thickness of $307 \mathrm{~nm}$ (Fig. S6, ESI $\dagger$ ). In the presence of the pump beam, the probe beam is amplified (Fig. S7, $\mathrm{ESI} \dagger$ ). The net material gain values at different temperatures and excitation power densities are shown in Fig. 5b-e. Clearly, the probe signal is amplified when passing through the excited films even at room temperature, and we can obtain optical amplifications in a broad spectral range of 1200-1625 nm, covering the whole telecommunication window of $\mathrm{O}(1260-1360 \mathrm{~nm})$, E (1360-1460 nm), and S (1460-1530 nm) bands, and even C (1530-1565 nm) and L (1565-1625 nm) bands. Benefiting from the improved PL emission at low temperatures, a net gain of $1.2 \times$ $10^{5} \mathrm{~cm}^{-1}$ at $1310 \mathrm{~nm}$ can be attained at $77 \mathrm{~K}$ with a power density as low as $0.38 \mathrm{~mW} \mathrm{~mm}^{-2}$. Previously, optical amplification has been realized in Bi-doped bulk materials (e.g., glasses) under high excitation power densities. ${ }^{4,32-34}$ We find that the maximum value achieved using our system is over five orders of magnitude larger than those achieved in Bi-doped bulk materials (Table S2, ESI $\dagger$ and Fig. 5). We also note that even at room temperature, the gain coefficient of the system is three orders of magnitude larger than those of bulk materials. The superior performance demonstrated here profits from the extremely high absorption coefficient on the order of $10^{4} \mathrm{~cm}^{-1}$ for the $\mathrm{MAPbX}_{3}$ semiconductor and relatively large QE at cryogenic temperatures (Table S3, ESI $\dagger$ ). ${ }^{24}$ To the best of our knowledge, this is the first evidence of light amplification in the telecommunication window for Bi-doped films and lead halide perovskite semiconductors. ${ }^{35-38}$ It is also noteworthy that Bi-doped $\mathrm{MAPbX}_{3}$ can be excited by photons with energies larger

than the bandgap energy, thus greatly relaxing the requirement of excitation sources for optical amplification. Generally, increasing bandwidth capacities while simultaneously reducing the number of power-hungry components in optical communication networks has proved challenging. However, the system we report here can realize NIR optical amplification under low excitation power densities, providing new opportunities in constructing energysaving devices for optical communication.

\section{Conclusions}

In summary, we have demonstrated that solution-processable, Bi-activated, high-quality $\mathrm{MAPbI}_{3}$ films can be adopted as a new gain medium operating in the whole telecommunication window. PDS measurement helps us ascertain that the observed NIR PL is associated with Bi-doping-induced structural defects. Furthermore, we found that the system shows strong NIR emission at a relatively low temperature that can be readily realized using inexpensive refrigeration setups such as liquid nitrogen cooling systems. Additionally, owing to the broad excitation band of this system, low-cost laser diodes or even light emitting diodes can be used as the excitation sources. ${ }^{30}$ Most importantly, we realized optical amplification in the whole telecommunication window, with the highest net gain ever reported, by using Bi-doped $\mathrm{MAPbI}_{3}$ films, which represents the first work on the attainment of such a performance among lead halide perovskites and Bi-doped photonic films. We expect our work to stimulate the development of more efficient luminescent system emitting in the NIR region via the extension of material systems studied. This work opens up exciting possibilities of using semiconducting perovskite materials as gain media for optical amplifiers and lasers operating in the telecommunication window.

\section{Acknowledgements}

Y. Zhou and Z.-J. Yong contributed equally to this work. This work is financially supported by the National Natural Science Foundation of China (11574225), Jiangsu Specially Appointed Professor program (SR10900214), Natural Science Foundation of Jiangsu Province for Young Scholars (BK20140336), and a project funded by the Priority Academic Program Development of Jiangsu Higher Education Institutions (PAPD). H.-T. Sun acknowledges financial support by the Open Fund of the State Key Laboratory of Luminescent Materials and Devices (South China University of Technology). A. Sadhanala would like to acknowledge the funding from EPSRC and India-UK APEX project. We would also like to thank Prof. Sir Richard Friend for the useful discussions.

\section{Notes and references}

1 J. Hecht, Nature, 2016, 536, 139-142.

2 L. K. Oxenlowe, Nat. Photonics, 2011, 5, 329-331.

3 G. A. Thomas, B. I. Shraiman, P. F. Glodis and M. J. Stephen, Nature, 2000, 404, 262-264. 
4 H. T. Sun, J. J. Zhou and J. R. Qiu, Prog. Mater. Sci., 2014, 64, $1-72$.

5 A. Kojima, K. Teshima, Y. Shirai and T. Miyasaka, J. Am. Chem. Soc., 2009, 131, 6050-6051.

6 M. M. Lee, J. Teuscher, T. Miyasaka, T. N. Murakami and H. J. Snaith, Science, 2012, 338, 643-647.

7 H.-S. Kim, I. Mora-Sero, V. Gonzalez-Pedro, F. FabregatSantiago, E. J. Juarez-Perez, N.-G. Park and J. Bisquert, Nat. Commun., 2013, 4, 2242.

8 W. Zhang, M. Saliba, D. T. Moore, S. K. Pathak, M. T. Horantner, T. Stergiopoulos, S. D. Stranks, G. E. Eperon, J. A. Alexander-Webber, A. Abate, A. Sadhanala, S. Yao, Y. Chen, R. H. Friend, L. A. Estroff, U. Wiesner and H. J. Snaith, Nat. Commun., 2015, 6, 6142.

9 Z. Tan, R. S. Moghaddam, M. Lai, P. Docampo, R. Higler, F. Deschler, M. Price, A. Sadhanala, L. M. Pazos, D. Credgington, F. Hanusch, T. Bein, H. J. Snaith and R. H. Friend, Nat. Nanotechnol., 2014, 9, 687-692.

10 A. Sadhanala, S. Ahmad, B. Zhao, N. Giesbrecht, P. M. Pearce, F. Deschler, R. L. Z. Hoye, K. C. Godel, T. Bein, P. Docampo, S. E. Dutton, M. F. L. De Volder and R. H. Friend, Nano Lett., 2015, 15, 6095-6101.

11 H. Cho, S.-H. Jeong, M.-H. Park, Y.-H. Kim, C. Wolf, C.-L. Lee, J. H. Heo, A. Sadhanala, N. Myoung, S. Yoo, S. H. Im, R. H. Friend and T.-W. Lee, Science, 2015, 350, 1222-1225.

12 J. Pan, L. N. Quan, Y. Zhao, W. Peng, B. Murali, S. P. Sarmah, M. Yuan, L. Sinatra, N. M. Alyami, J. Liu, E. Yassitepe, Z. Yang, O. Voznyy, R. Comin, M. N. Hedhili, O. F. Mohammed, Z. H. Lu, D. H. Kim, E. H. Sargent and O. M. Bakr, Adv. Mater., 2016, 28, 8718-8725.

13 M. Yuan, L. N. Quan, R. Comin, G. Walters, R. Sabatini, O. Voznyy, S. Hoogland, Y. Zhao, E. M. Beauregard, P. Kanjanaboos, Z. Lu, D. H. Kim and E. H. Sargent, Nat. Nanotechnol., 2016, 11, 872-877.

14 Z. J. Ning, X. W. Gong, R. Comin, G. Walters, F. J. Fan, O. Voznyy, E. Yassitepe, A. Buin, S. Hoogland and E. H. Sargent, Nature, 2015, 523, 324-328.

15 S. Kondo, T. Saito, H. Asada and H. Nakagawa, Mater. Sci. Eng., B, 2007, 137, 156-161.

16 G. Xing, N. Mathews, S. Lim, N. Yantara, X. Liu, D. Sabba, M. Grätzel, S. Mhaisalkar and T. C. Sum, Nat. Mater., 2014, 13, 476-480.

17 F. Deschler, M. Price, S. Pathak, L. E. Klintberg, D. Jarausch, R. Higler, S. Huttner, T. Leijtens, S. D. Stranks, H. J. Snaith, M. Atature, R. T. Phillips and R. H. Friend, J. Phys. Chem. Lett., 2014, 5, 1421-1426.

18 S. Yakunin, L. Protesescu, F. Krieg, M. I. Bodnarchuk, G. Nedelcu, M. Humer, G. D. Luca, M. Fiebig, W. Heiss and M. V. Kovalenko, Nat. Commun., 2015, 6, 8056.
19 H. Zhu, Y. Fu, F. Meng, X. Wu, Z. Gong, Q. Ding, M. V. Gustafsson, M. T. Trinh, S. Jin and X.-Y. Zhu, Nat. Mater., 2015, 14, 636-642.

20 Y. Fang, Q. Dong, Y. Shao, Y. Yuan and J. Huang, Nat. Photonics, 2015, 9, 679-686.

21 M. I. Saidaminov, M. A. Haque, M. Savoie, A. L. Abdelhady, N. Cho, I. Dursun, U. Buttner, E. Alarousu, T. Wu and O. M. Bakr, Adv. Mater., 2016, 28, 8144-8149.

22 F. Hu, H. Zhang, C. Sun, C. Yin, B. Lv, C. Zhang, W. W. Yu, X. Wang, Y. Zhang and M. Xiao, ACS Nano, 2015, 9, 12410-12416.

23 D. Q. Chen, Z. Wan, X. Chen, Y. Yuan and J. Zhong, J. Mater. Chem. C, 2016, 4, 10646-10653.

24 J.-H. Im, I.-H. Jang, N. Pellet, M. Grätzel and N.-G. Park, Nat. Nanotechnol., 2014, 9, 927-932.

25 B. R. Sutherland and E. H. Sargent, Nat. Photonics, 2016, 10, 295-302.

26 D. Shi, V. Adinolfi, R. Comin, M. Yuan, E. Alarousu, A. Buin, Y. Chen, S. Hoogland, A. Rothenberger, K. Katsiev, Y. Losovyj, X. Zhang, P. A. Dowben, O. F. Mohammed, E. H. Sargent and O. M. Bakr, Science, 2015, 347, 519-522.

27 H. Fang, R. Raissa, M. Abdu-Aguye, S. Adjokatse, G. R. Blake, J. Even and M. A. Loi, Adv. Funct. Mater., 2015, 25, 2378-2385.

28 X. Li, D. Bi, C. Yi, J.-D. Décoppet, J. Luo, S. M. Zakeeruddin, A. Hagfeldt and M. Grätzel, Science, 2016, 353, 58-62.

29 A. L. Abdelhady, M. I. Saidaminov, B. Murali, V. Adinolfi, O. Voznyy, K. Katsiev, E. Alarousu, R. Comin, I. Dursun, L. Sinatra, E. H. Sargent, O. F. Mohammed and O. M. Bakr, J. Phys. Chem. Lett., 2016, 7, 295-301.

30 Y. Zhou, Z.-J. Yong, K.-C. Zhang, B.-M. Liu, Z.-W. Wang, J.-S. Hou, Y.-Z. Fang, Y. Zhou, H.-T. Sun and B. Song, J. Phys. Chem. Lett., 2016, 7, 2735-2741.

31 L. Pavesi, L. Dal Negro, C. Mazzoleni, G. Franzo and F. Priolo, Nature, 2000, 408, 440-444.

32 Y. Fujimoto and M. Nakatsuka, Appl. Phys. Lett., 2003, 82, 3325-3326.

33 S. Zhou, H. Dong, H. Zeng, J. Hao, J. Chen and J. R. Qiu, J. Appl. Phys., 2008, 103, 103532.

34 J. Ruan, E. Wu, H. P. Zeng, S. F. Zhou, G. Lakshminarayana and J. R. Qiu, Appl. Phys. Lett., 2008, 92, 101121.

35 H.-T. Sun, F. Shimaoka, Y. Miwa, J. Ruan, M. Fujii, J. Qiu and S. Hayashi, Opt. Lett., 2010, 35, 2215-2217.

36 Y. Miwa, H.-T. Sun, K. Imakita, M. Fujii, Y. Teng, J. R. Qiu, Y. Sakka and S. Hayashi, Opt. Lett., 2011, 36, 4221-4223.

37 M. Fujii, S. Morimoto, S. Kitano, K. Imakita, J. R. Qiu and H.-T. Sun, Opt. Lett., 2013, 38, 4224-4227.

38 B. Xu, J. H. Hao, S. F. Zhou and J. R. Qiu, Opt. Express, 2013, 21, 18532-18537. 